\title{
3D-Quantification using an interleaved Look-Locker acquisition sequence with T2-prep pulse (3D-QALAS)
}

\author{
Sofia Kvernby ${ }^{1,2^{*}}$, Marcel Warntjes ${ }^{1,2}$, Carl-Johan Carlhäll ${ }^{1,2}$, Jan Engvall ${ }^{1,2}$, Tino Ebbers ${ }^{1,2}$ \\ From 17th Annual SCMR Scientific Sessions \\ New Orleans, LA, USA. 16-19 January 2014
}

\section{Background}

Quantification of the longitudinal- and transverse relaxation time in the myocardium has shown to provide important information in cardiac diagnostics. Several methods are currently available for cardiac relaxation time mapping but they generally demand a long breath hold to measure either $\mathrm{T} 1$ or $\mathrm{T} 2$ in a single $2 \mathrm{D}$ slice. In order to make quantification of relaxation parameters in the myocardium clinically applicable, the methods must be improved in speed and coverage. In this work we propose a novel method for 3D interleaved $\mathrm{T} 1$ and T2 quantification of the whole left ventricular myocardium within one single breath hold.

\section{Methods}

The sequence is based on a 3D spoiled Turbo Field Echo sequence using inversion recovery with interleaved T2 preparation and is referred to as 3D-QALAS (3D-quantification using an interleaved Look-Locker acquisition sequence with T2 preparation pulse). Quantification of both T1 and T2 in a volume of 13 slices with a resolution of $2.0 \times 2.0 \times 6.0 \mathrm{~mm}$ is obtained. The acquisition time is restricted to $230 \mathrm{~ms}$ during end diastole to avoid artifacts from cardiac motion. Data acquisition is performed once after a T2 preparation pulse and four times after a T1 sensitizing inversion pulse ( 5 heartbeats). This acquisition scheme is repeated three times to sample the 3D k-space, resulting in a breath hold of 15 heartbeats. The sequence was validated in both phantoms and healthy volunteers using a Philips Ingenia 3T scanner. The effect of different heart rates, flip angles and deliberately applied arrhythmias were simulated and investigated in phantoms.

${ }^{1}$ Linköping University, Linköping, Sweden

Full list of author information is available at the end of the article

\section{Results}

Relaxation times in phantoms correlate well with reference methods, Inversion Recovery for $\mathrm{T} 1(\mathrm{R}=0,999)$ and Multi Echo for T2(R = 0,960). Quantification of T1 and T2 using 3D QALAS showed no dependence on heart rate, flip angle or arrhythmia within the clinically relevant range. In vitro $\mathrm{T} 1$ measurements with $3 \mathrm{D}$ QALAS for different heart rates, which has shown to be challenging for other methods, are shown in Figure 1. In Figure 2, T1- and T2 maps are shown for a healthy volunteer. The mean native myocardial $\mathrm{T} 1$ and $\mathrm{T} 2$ values in this volunteer were $1085 \pm 39$ ms (mean \pm $\mathrm{SD})$ and $52 \pm 5 \mathrm{~ms}$ which is in accordance with previous results (Piechnik S. et Al, 2010, JCMR; Shivraman G. et $\mathrm{Al}, 2009$, JCMR).

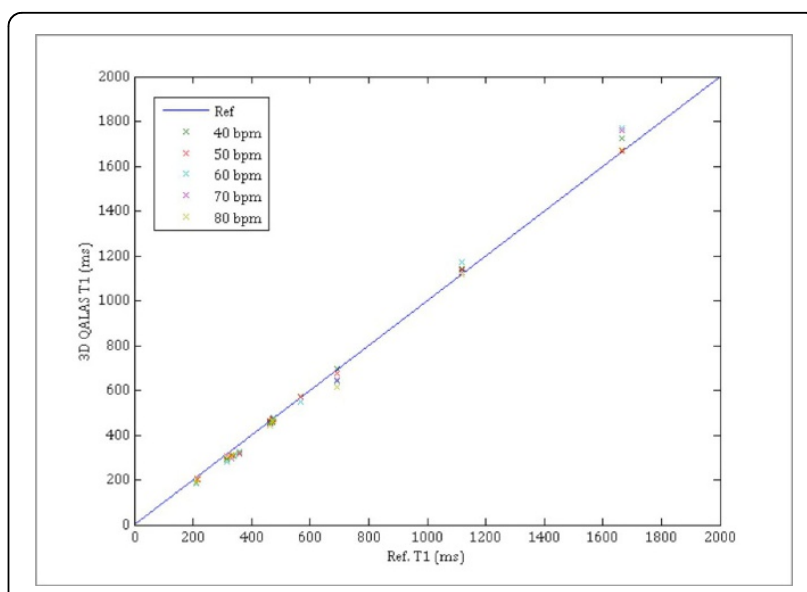

Figure 1 Measurements of T1 in phantoms with 3D QALAS and the corresponding reference $\mathrm{T} 1$ for specific simulated heart rates. 


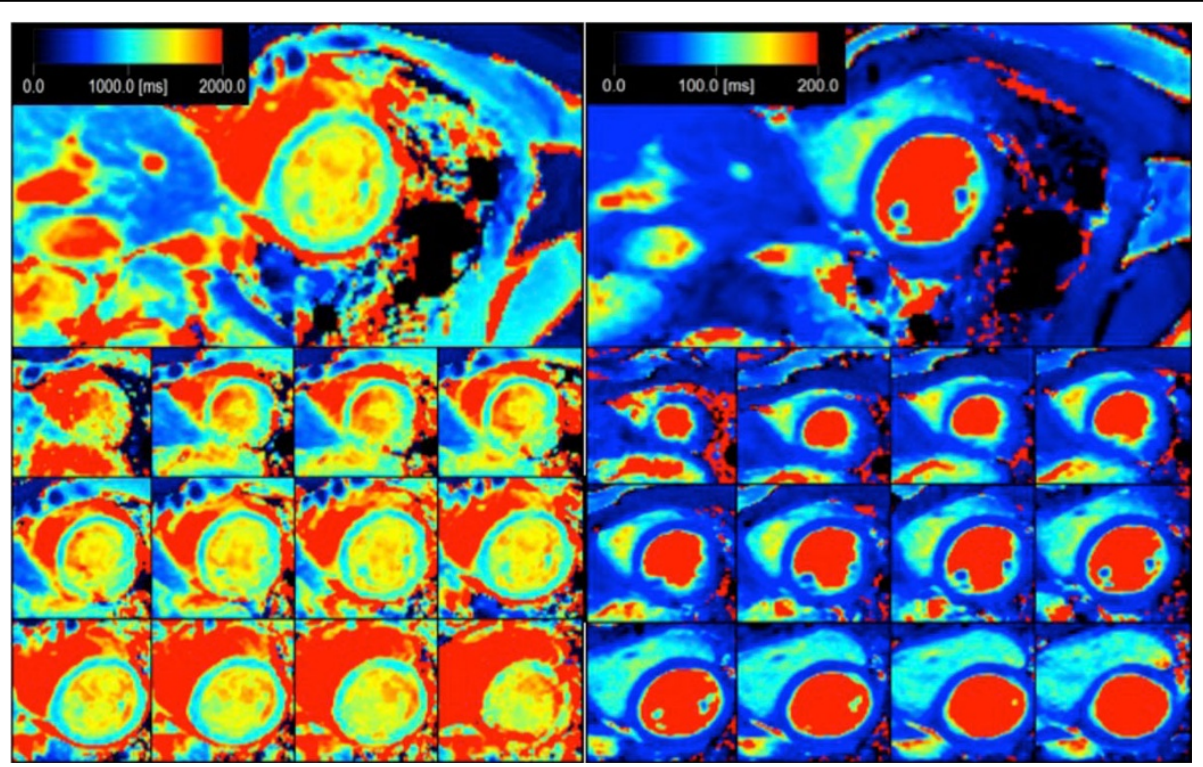

Figure 2 3D QALAS T1 maps (left) and T2 maps (right) of 13 slices for a healthy volunteer

\section{Conclusions}

3D QALAS allows quantification of both $\mathrm{T} 1$ and $\mathrm{T} 2$ in the whole left ventricular myocardium within one breath hold, making $\mathrm{T} 1$ and $\mathrm{T} 2$ quantification clinically applicable to a broader spectrum of diseases.

\section{Funding}

Swedish Research Council and Swedish Heart-Lung Foundation.

\section{Authors' details}

'Linköping University, Linköping, Sweden. ${ }^{2}$ Center for Medical Image Science and Visualization (CMIV), Linköping, Sweden.

Published: 16 January 2014

doi:10.1186/1532-429X-16-S1-082

Cite this article as: Kvernby et al: 3D-Quantification using an interleaved Look-Locker acquisition sequence with T2-prep pulse (3D-QALAS). Journal of Cardiovascular Magnetic Resonance 2014 16(Suppl 1):O82.
Submit your next manuscript to BioMed Central and take full advantage of:

- Convenient online submission

- Thorough peer review

- No space constraints or color figure charges

- Immediate publication on acceptance

- Inclusion in PubMed, CAS, Scopus and Google Scholar

- Research which is freely available for redistribution 